\title{
Modern Analytical Electrochemistry: Fundamentals, Experimental Techniques, and Applications
}

\author{
Bengi Uslu, ${ }^{1}$ Hassan Y. Aboul-Enein, ${ }^{2}$ and Sibel A. Ozkan ${ }^{1}$ \\ ${ }^{1}$ Department of Analytical Chemistry, Faculty of Pharmacy, Ankara University, Tandogan, 06100 Ankara, Turkey \\ ${ }^{2}$ Pharmaceutical and Medicinal Chemistry Department, National Research Centre, Cairo 12311, Egypt
}

Correspondence should be addressed to Bengi Uslu, buslu@pharmacy.ankara.edu.tr

Received 16 November 2011; Accepted 16 November 2011

Copyright (c) 2011 Bengi Uslu et al. This is an open access article distributed under the Creative Commons Attribution License, which permits unrestricted use, distribution, and reproduction in any medium, provided the original work is properly cited.

Such use of electrical measurements for analytical purposes has found a vast range of applications, including environmental monitoring, drug and biomedical analysis. Advances in the 1980s and 1990s-including the development of ultramicroelectrodes, the coupling of biological components and electrochemical transducers, the development of ultratrace voltammetric techniques (such as differential pulse voltammetry, square wave voltammetry, stripping voltammetry, etc.) or the use of single-walled and multiwalled carbon nanotubes in scanning probe microscopies, and the microfabrication of molecular devices or efficient flow detectors-have led to a substantial increase in the popularity of electroanalysis and to its expansion into new phases environments.

The use of electrochemical methods to gain key information about drug molecules and their mechanism of action is getting one of the important ways in drug discovery. It should be noticed that many vital physiological processes are depending on oxidoreduction reactions. So, it is easy to find connections between electrochemical and biological reactions regarding electron transfer pathways.

Progress in genomics and particularly in the Human Genome Project in that time stimulated enormous interest in new methods capable to unravel the genetic information stored in the nucleotide sequence of DNA. Only after the construction of DNA chips with optical detection, the attempts to developed DNA chips with electrochemical detection have become popular among electrochemists. Since the middle of 1990s, the electrochemistry of nucleic acids and the development of DNA sensors have become a booming field involving large number of laboratories all over the world. Electrochemical DNA biosensors enable the study of the interaction of DNA immobilized on the electrode surface with analytes in solution. The investigation based on DNA interactions has great importance in understanding the mechanism of action of many drug compound and complexes, designing of new DNA-drug biosensors, and screening of the drugs in vitro. Electrochemical investigations of nucleic acid binding molecules-DNA interactions can provide a useful complement to the spectroscopic techniques and yield information about the mechanism at intercalation and the confirmation of anti-HIV-DNA adduct.

Analytical electrochemists play important roles in monitoring the drug and some metals in their dosage forms, biological samples, and environmental samples. From the viewpoints mentioned above, the title of this special issue "Modern analytical electrochemistry: fundamentals, experimental techniques, and applications" was chosen so as to ask electrochemists to appreciate their great roles in chemistry science. This special issue features 5 review and 20 research articles. In this issue: Recent development of electroanalytical techniques such as differential pulse, square wave and their pharmaceutical and environmental applications are presented. It mainly contains current progresses in modified electrodes, DNA biosensors, sensors and their applications to the pharmaceuticals and biological samples, and new electrode materials such as carbon nanotubes and borondoped diamond.

The recent development of modern analytical chemistry applications, evaluation of electroanalytical methods are focused by key topics in drug and environmental analysis by assessment of the distinguished authors of this special issue. Thus, we expect this special issue will assist readers to find out new information and to encourage them to contribute 
more to recent development on drug and environmental analysis using different electroanalytical methods.

The purpose of this special issue will be to serve as a guide to what electroanalytical methods bring to chemistry as well as briefly review their role in drug or environmental samples and the new developments and validation of assay methods of pharmaceutical active components in their dosage forms or in other samples. We hope that the reader will find a number of topics of interest and that additional new ideas will emerge from this special issue.

\section{Acknowledgment}

We would like to thank to all of the authors for their excellent contributions and the Editorial Board members of International Journal of Electrochemistry for their kind invitations to act as guest editors for this special issue.

Bengi Uslu Hassan Y. Aboul-Enein Sibel A. Ozkan 


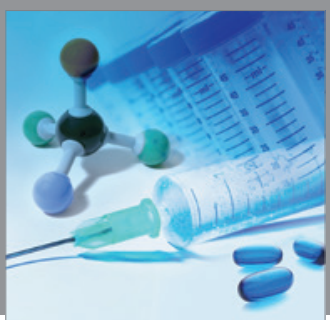

International Journal of

Medicinal Chemistry

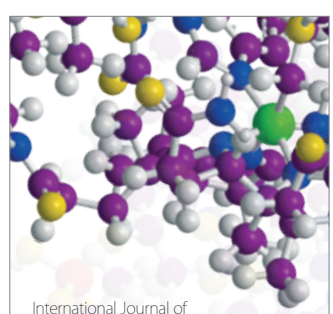

Carbohydrate Chemistry

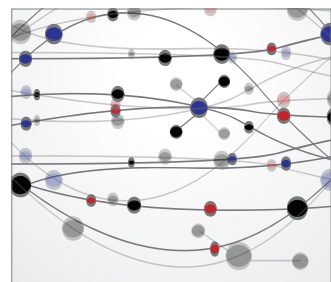

The Scientific World Journal
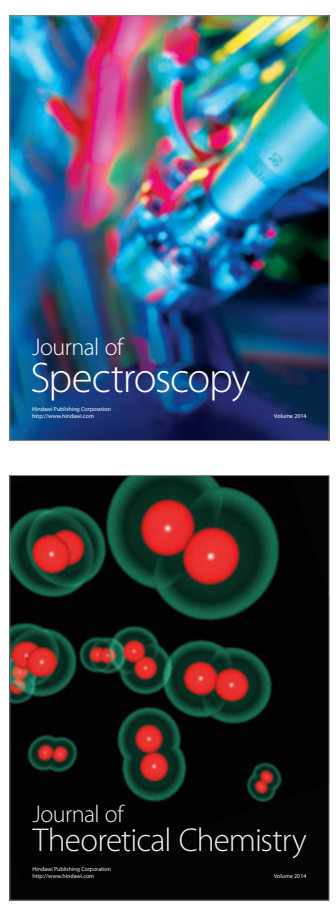
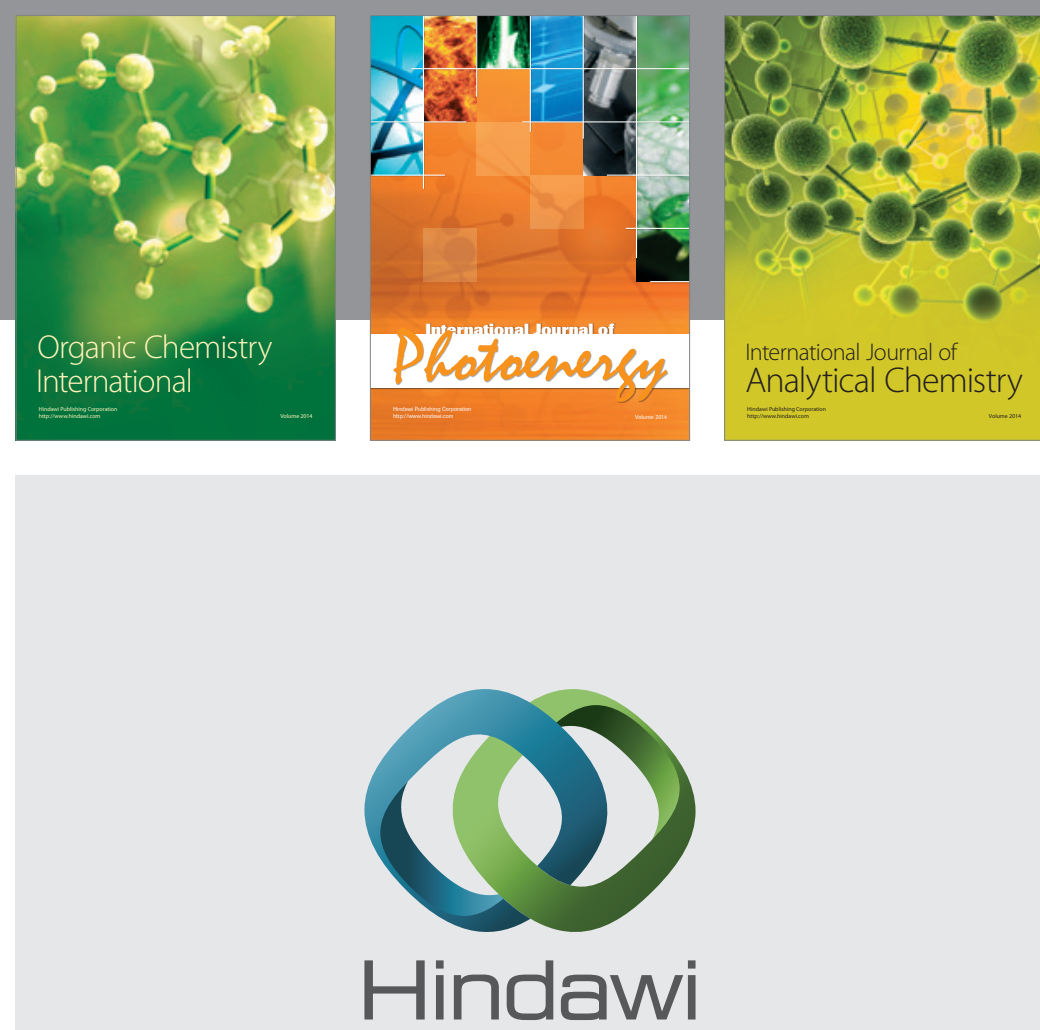

Submit your manuscripts at

http://www.hindawi.com
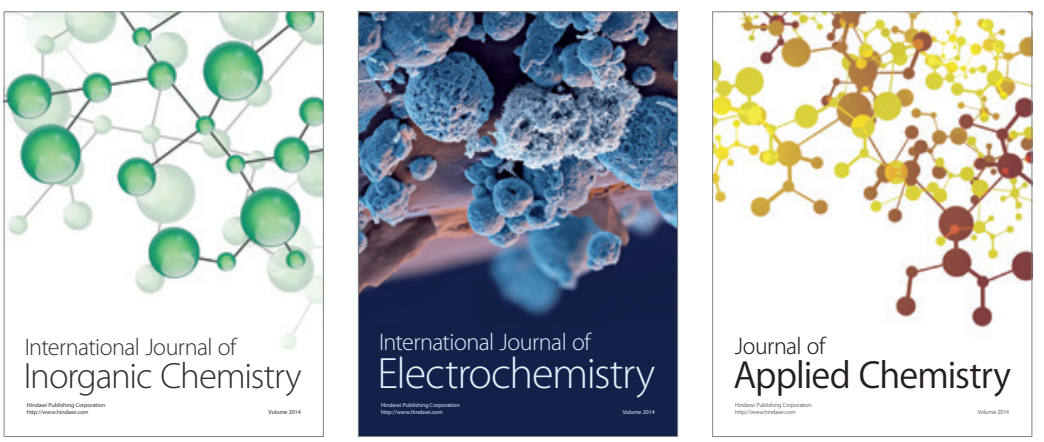

Journal of

Applied Chemistry
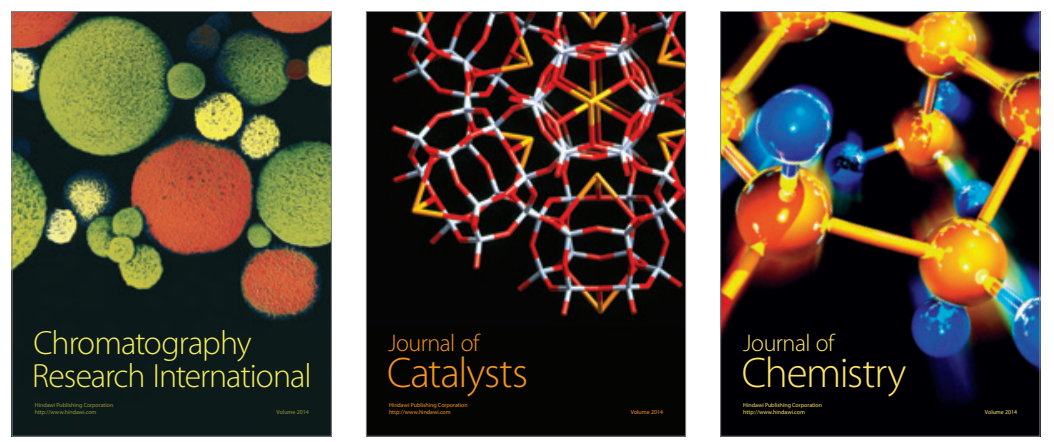
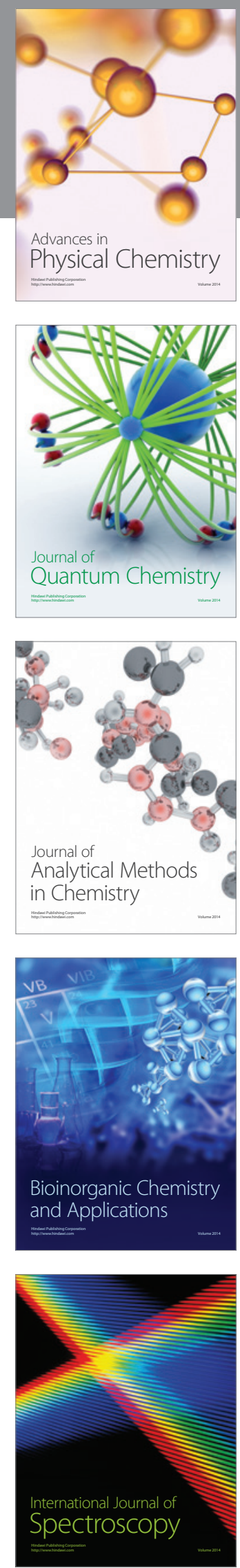